\title{
Enzyme-Functionalized Cellulose Beads as a Promising Antimicrobial Material
}

Davide Califano $^{\mathrm{a}, \mathrm{b}}$, Bethany Lee Patenall ${ }^{\mathrm{a}, \mathrm{b}}$, Marco A. S. Kadowaki ${ }^{\mathrm{a}}$, Davide Mattia ${ }^{\mathrm{c}}$, Janet L. Scott $^{\mathrm{a}, \mathrm{b}}$, Karen J. Edler*a

a. Department of Chemistry, University of Bath, Claverton Down, Bath, UK.

b. Centre for Sustainable Chemical Technologies, University of Bath, Claverton Down, Bath, UK.

c. Department of Chemical engineering, University of Bath, Claverton Down, Bath, UK. 
Figure ESI 1: Beads size upon periodate oxidation. The beads diameter significantly decreased in cellulose beads with a higher degree of oxidation suggesting a structural rearrangement of the polymer network. Error bars represent the standard deviation obtained from $(\mathrm{n}=30)$.

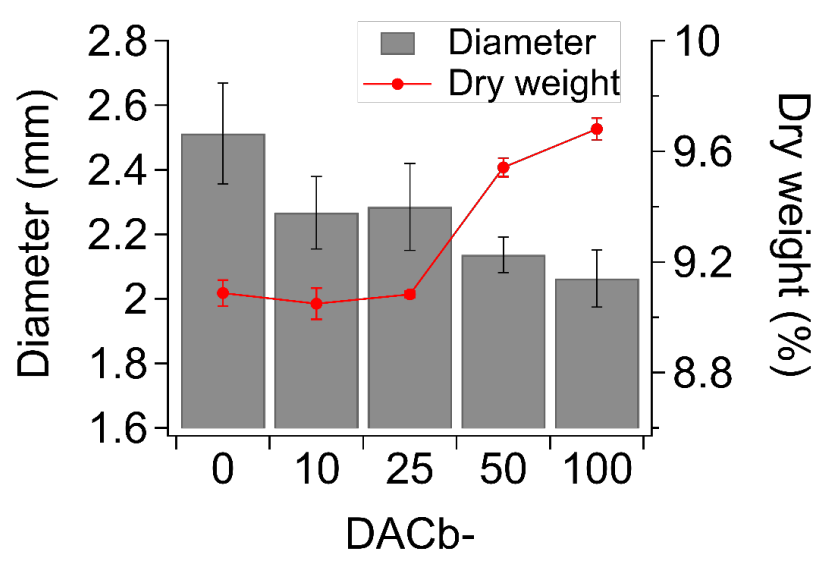


Figure ESI 2: Uniaxial deformation test. The plots from (a) to (e) show the resistance to compression of DACb-0, DACb-10, DACb-25, DACb-50 and DACb-100 respectively after exposure at pH 6 (blue), 8 (green) and 10 (orange). All curves are characterized by an initial steady increase, followed by a sharp peak of the axial force over the strain which represent the elastic region and a strain-hardening region.
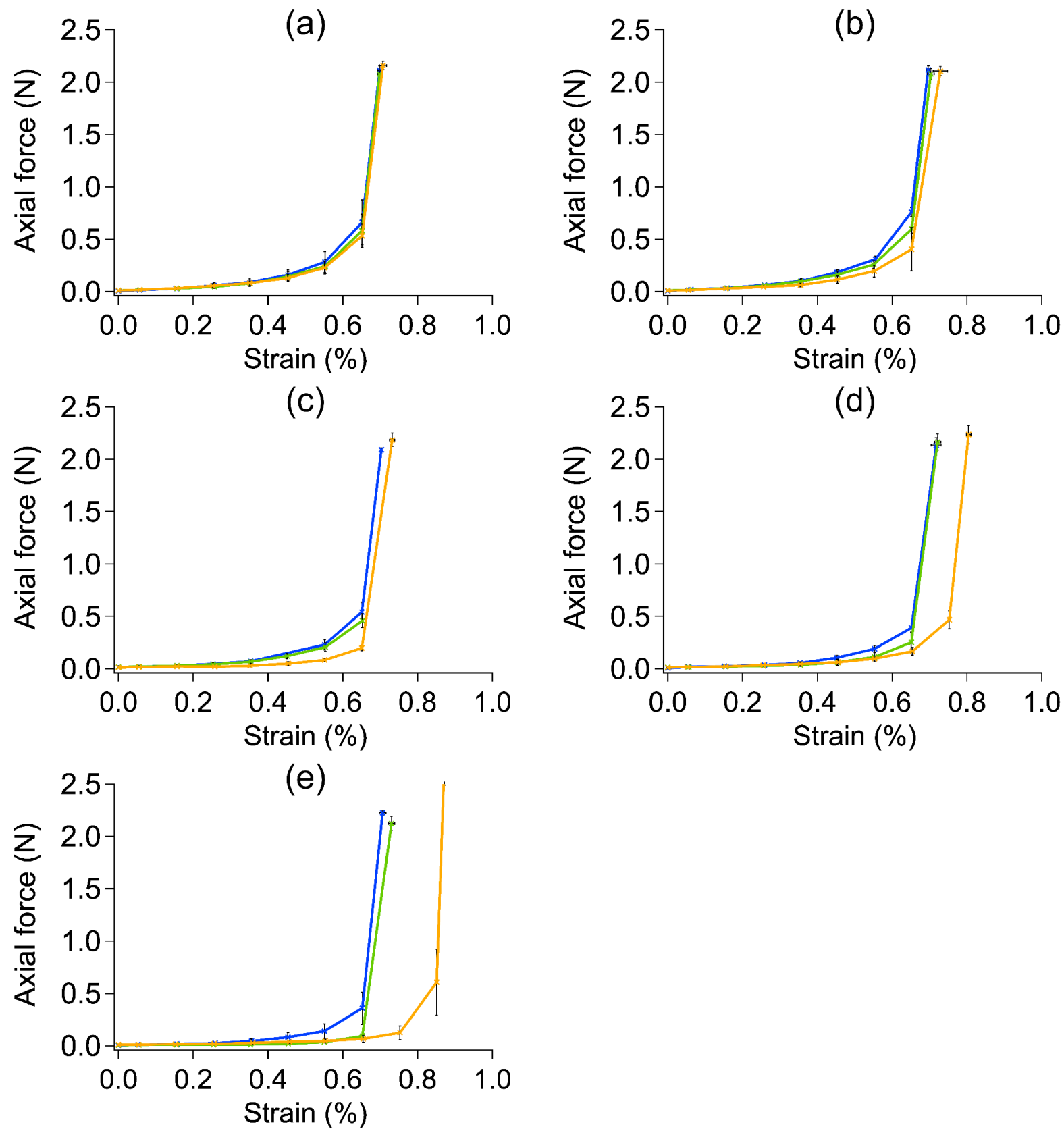
Figure ESI 3: Scanning electron micrographs of DACb-100 functionalized at $\mathrm{pH} 10$. (a) The bead shows multiple fractures upon freeze-drying indicating structural damage of the cellulose network. (b) Structural damage of the internal structure and the outer layer bead of a single bead. (c) A magnified picture of the bead core showing the brittleness of the internal structure.

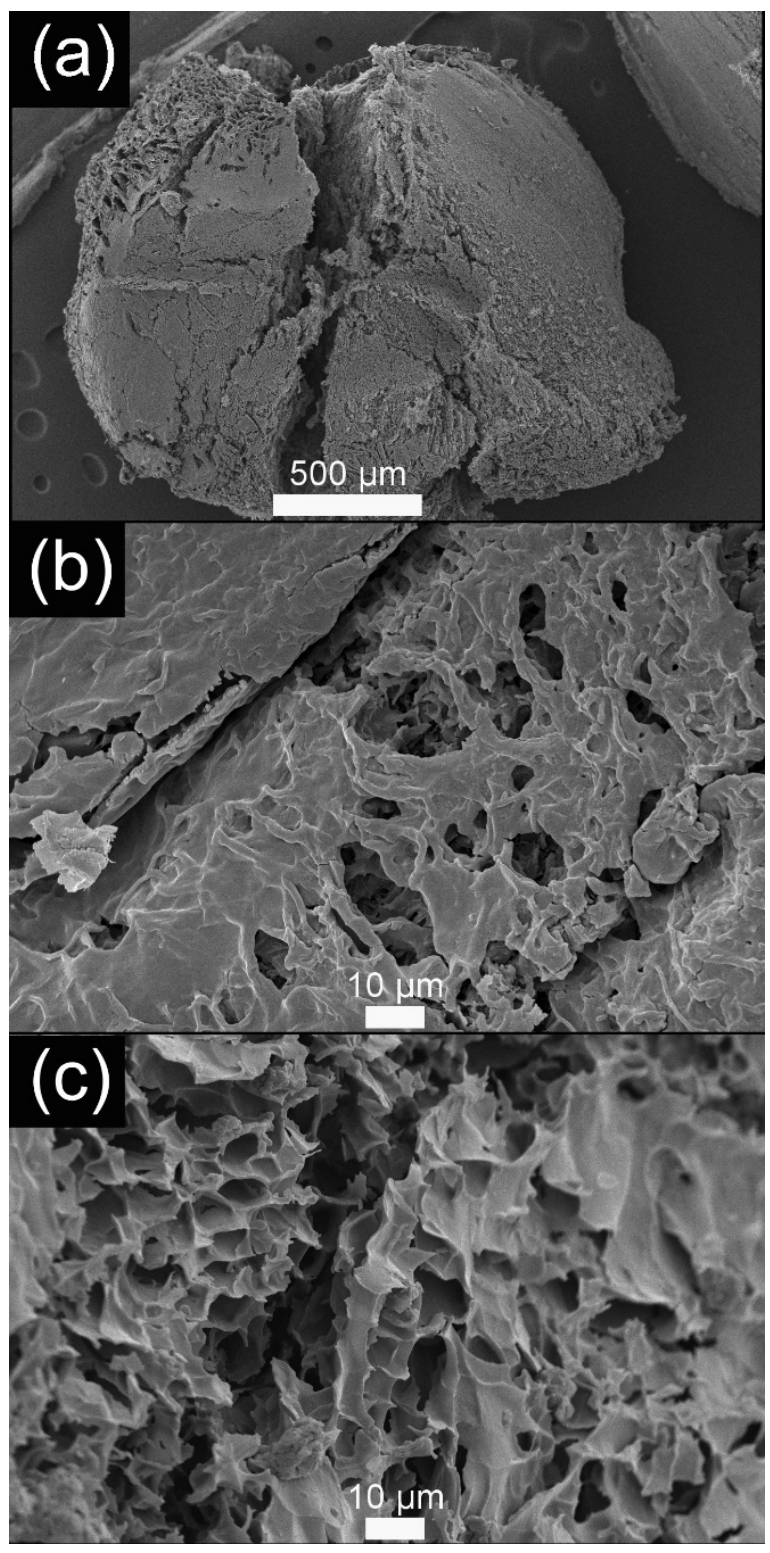


Figure ESI 4: Kinetic curves of DACb at different degree of oxidation and functionalized using different $\mathrm{pH}$. All $\mathrm{H}_{2} \mathrm{O}_{2}$ kinetic curves show a linear growth in the first $2.5 \mathrm{~h}$ followed by a flattening and a saturation point. The plots from (a) to (e) display the $\mathrm{H}_{2} \mathrm{O}_{2}$ kinetics of DACb- 0 , DACb-10, DACb-25,

DACb-50 and DACb-100 respectively. Line colors represent DACb functionalized at different, blue at $\mathrm{pH}$ 6 , green at $\mathrm{pH} 8$ and orange at $\mathrm{pH} 10$. The shadows represent the standard deviation $(\mathrm{n}=3)$.
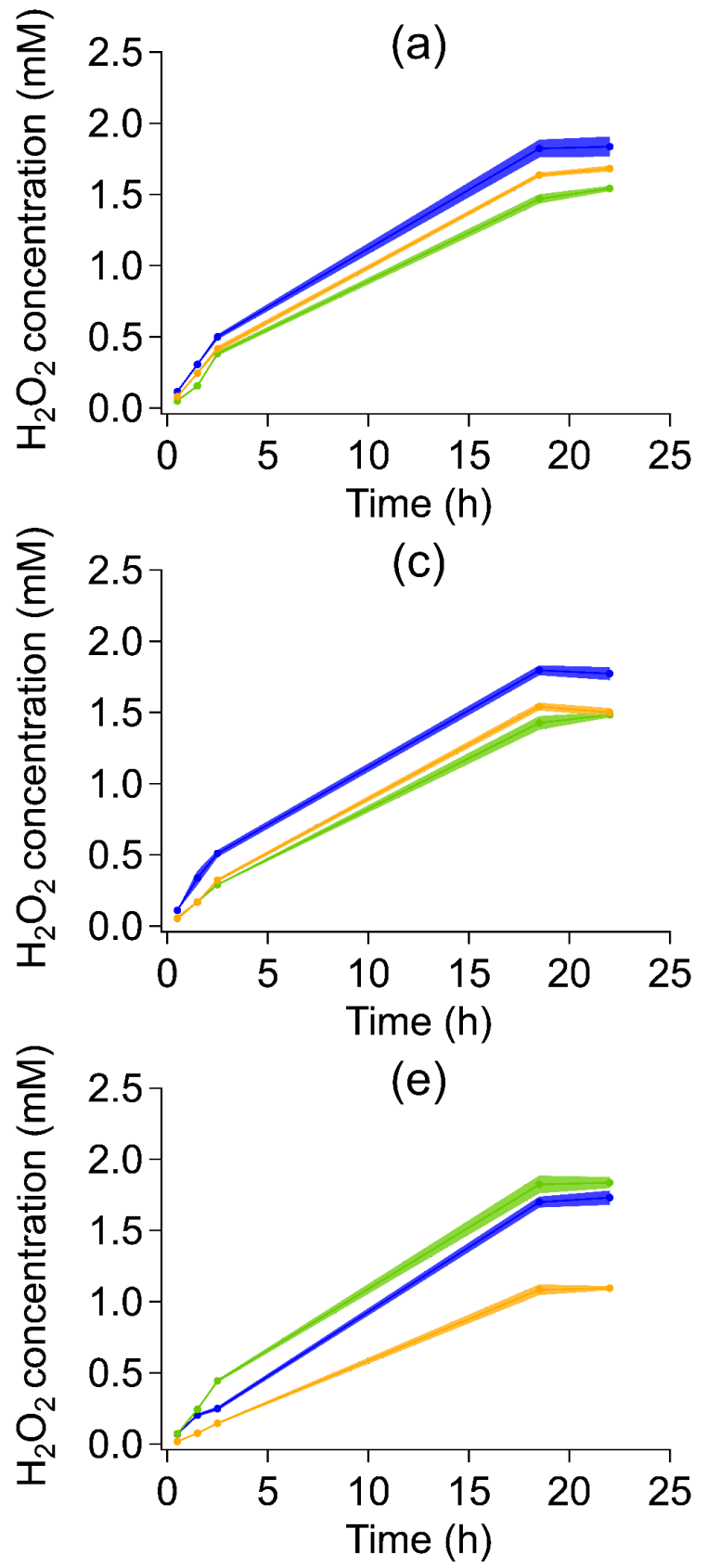

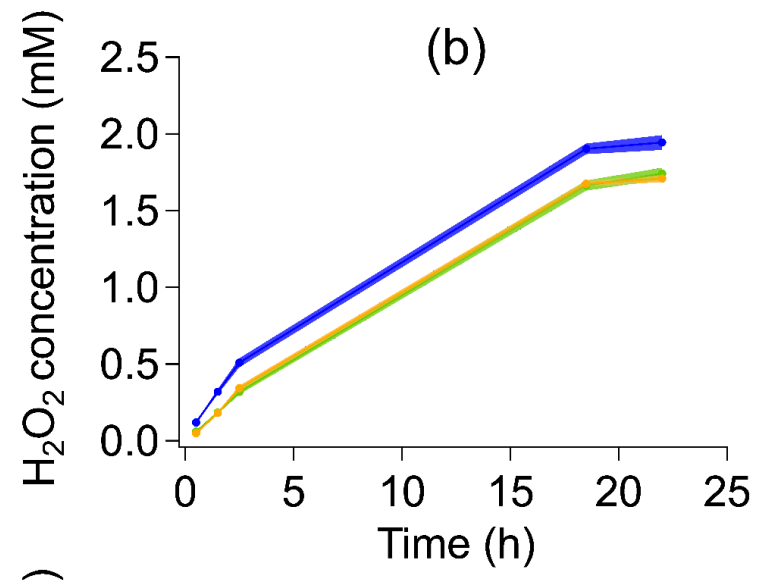

(d)

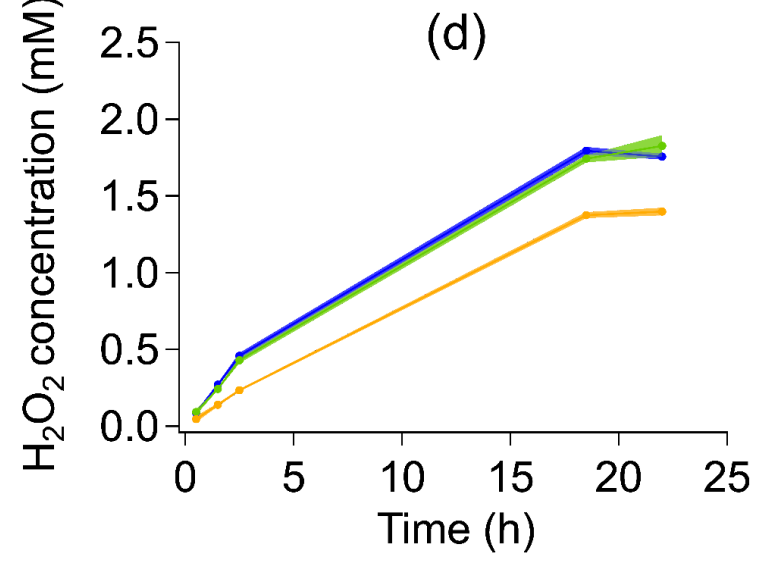


Figure ESI 5: Functionalized beads $\mathrm{H}_{2} \mathrm{O}_{2}$ release in a larger volume. The release of $\mathrm{H}_{2} \mathrm{O}_{2}$ from the functionalized beads increased linearly confirming that the GOx activity is not inhibited when exposed to lower concentrations of $\mathrm{H}_{2} \mathrm{O}_{2}$ compared those reached in smaller volumes $(\approx 2 \mathrm{mM})$. The shadows represent the standard deviation $(n=3)$.

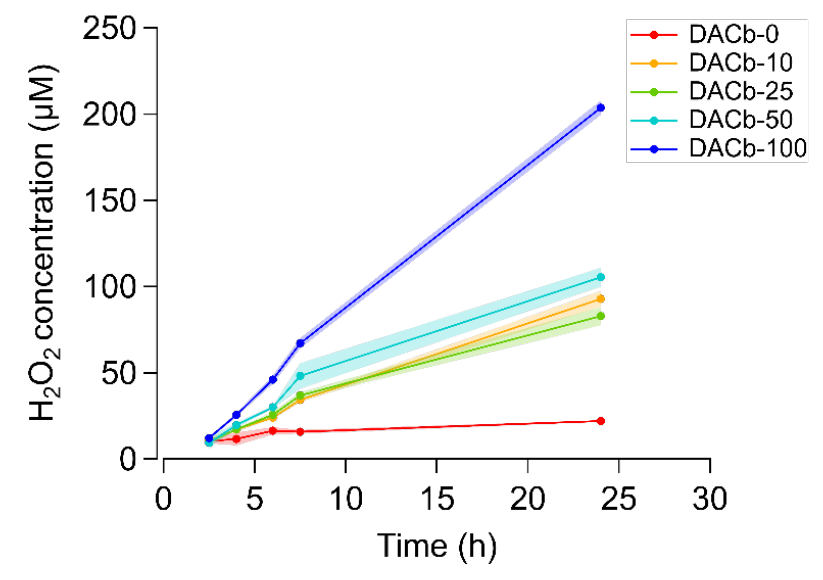


Figure ESI 6: Dose-dependent antimicrobial response of functionalized beads of the agar diffusion test.

The control beads did not contain GOx while the other beads where oxidized DACb-100 functionalized with GOx at pH 6. All beads were incubated with a buffered solution (phosphate buffer, pH 6, 0.1M) containing $1 \mathrm{wt} . \%$ glucose.

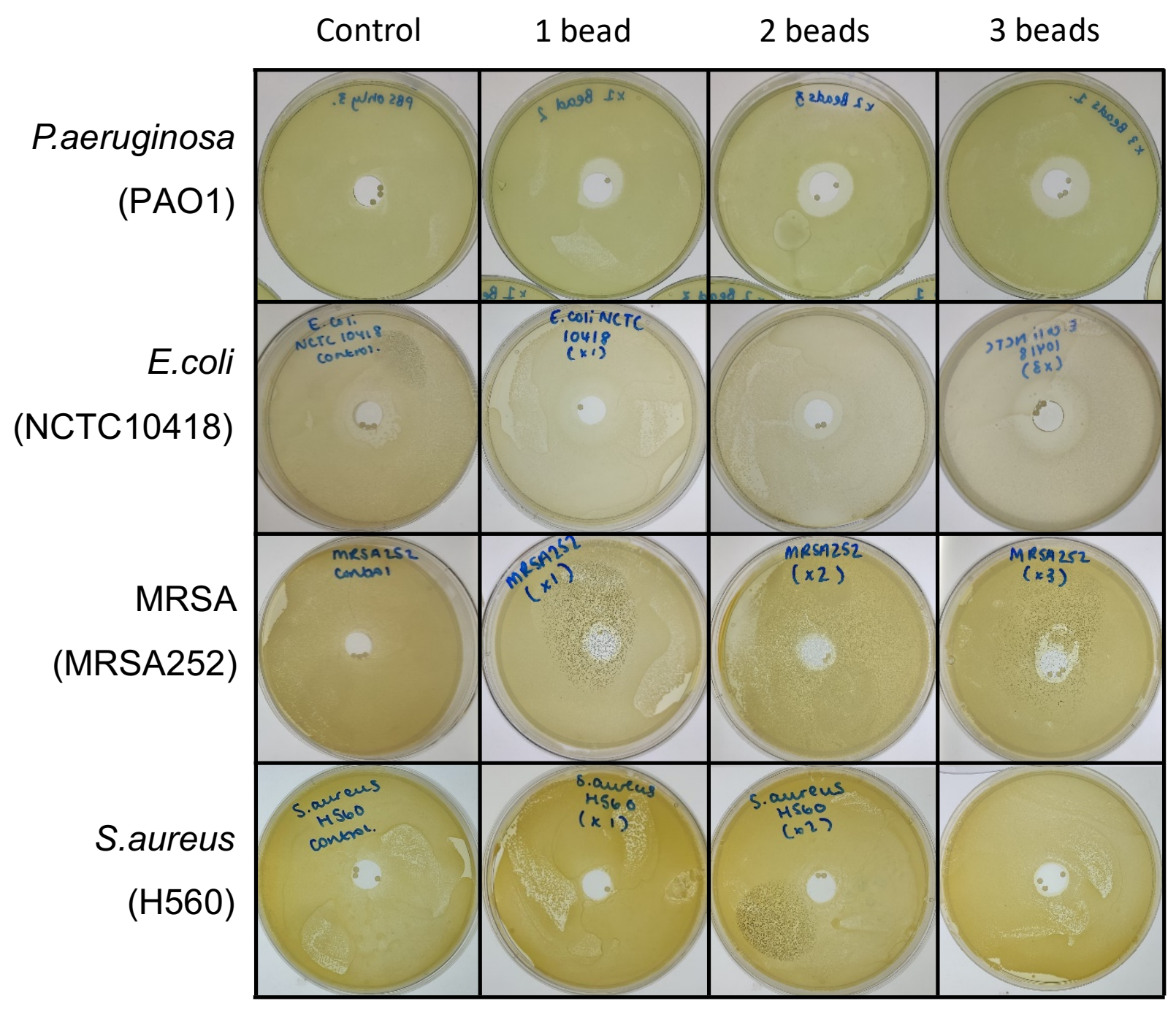

